\title{
CADM1/TSLC1 inhibits melanoma cell line A375 invasion through the suppression of matrix metalloproteinases
}

\author{
YAN YOU ${ }^{*}$, JINFENG ZHANG $^{2 *}$, YULIAN LI ${ }^{3}$, YUZHEN LI', \\ GUANGYUE $\mathrm{SHI}^{5}$, LANGJUAN MA ${ }^{4}$ and HAIYING WEI ${ }^{6}$
}

\author{
Departments of ${ }^{1}$ Dermatology and ${ }^{2}$ Thoracic Surgery, Third Affiliated Hospital, Harbin Medical University; \\ ${ }^{3}$ Education Administration Office, Third Affiliated Hospital, Harbin Medical University, Harbin, Heilongjiang 150081; \\ ${ }^{4}$ Department of Dermatology, Second Affiliated Hospital, Harbin Medical University, Harbin, Heilongjiang 150086; \\ ${ }^{5}$ Department of Oncology, Third Affiliated Hospital; ${ }^{6}$ Department of Ophthalmology, First Affiliated Hospital, \\ Harbin Medical University, Harbin, Heilongjiang 150081, P.R. China
}

Received October 23, 2013; Accepted May 14, 2014

DOI: $10.3892 / \mathrm{mmr} .2014 .2556$

\begin{abstract}
Increasing evidence has demonstrated that cell adhesion molecule 1/tumor suppressor in lung cancer 1 (CADM1/TSLC1) is crucially implicated in various biological processes, including proliferation, apoptosis, and invasion during tumorigenesis and development. However, the mechanism underlying its suppression of tumor growth and metastasis in melanoma remains elusive. The aim of the present study was to examine if CADM1/TSLC1 was able to induce growth suppression in melanoma. The plasmid pcDNA3.1-CADM1/TSLC1 was transfected into A375 cells (a human melanoma cell line). The expression of CADM1/TSLC1 in the transfected cells was determined by RT-PCR and western blotting analysis. Cell growth was measured by an 3-(4,5-dimethylthiazol-2-yl)-2,5-diphenyl-tetrazolium bromide assay and cell apoptosis was determined by flow cytometry, while a transwell assay was utilized to measure the ability of invasion. The expression of MMP-2 and MMP-9 in the transfected cells was determined by western blotting analysis. RT-PCR and western blotting revealed that in pcDNA3.1-CADM1/TSLC1 the protein expression of CADM1/TSLC1 protein was higher than in the pcDNA3.1 and A375 cells. The expression of MMP-2 and MMP-9 was lower in the pcDNA3.1-CADM1/TSLC1 than that in the pcDNA3.1 and A375 cells. The growth of CADM1/TSLC1-transfected cells was significantly suppressed in vitro and the ability
\end{abstract}

Correspondence to: Professor Yan You, Department of Dermatology, Third Affiliated Hospital, Harbin Medical University, 150 Haping Road, Harbin, Heilongjiang 150081, P.R. China

E-mail: yyxy1999@sina.com

*Contributed equally

Key words: cell adhesion molecule 1, tumor suppressor in lung cancer-1, matrix metalloproteinase-9, matrix metalloproteinase-2, proliferation, invasion, apoptosis of invasion was also reduced, CADM1/TSLC1 was able to induce cell apoptosis. Furthermore, CADM1/TSLC1 was an anti-invasive gene, the overexpression of which inhibited the invasion of A375 cells. This inhibition may be due to the suppression of the MMP-2 and MMP-9 expression, which is relative to tumor metastasis and progression.

\section{Introduction}

Cell adhesion molecule 1/tumor suppressor in lung cancer-1 (CADM1/TSLC1) is a tumor suppressor gene that was first identified by Murakami et al in lung carcinoma (1). Murakami et al observed that loss of heterozygosity ( $\mathrm{LOH}$ ) on chromosome 11q23 occurred in patients with non-small cell lung carcinoma and found it had growth suppression effects on the tumor cells. Therefore, the gene was named tumor suppressor in lung carcinoma-1 (TSLC1).

Loss or reduction of CADM1/TSLC1 expression was frequently observed and demonstrated to be involved in the progression and metastasis of a growing number of different tumor types, including lung cancer (2), gastric cancer (3), T-cell leukemia (4), ovarian carcinoma (5), pancreatic cancer (6) and breast cancer (7). In our previous study (8), it was demonstrated that silencing of CADM1/TSLC1 in melanoma is consistent with promoter methylation, and that the incidence of the loss of expression and methylation of CADM1/TSLC1 significantly increased as the tumor stage advanced. The present study focuses on the functional role of CADM1/TSLC1 in the tumorigenesis of melanoma. To examine the possible growth-suppressive activity of CADM1/TSLC in melanoma, a stable CADM1/TSLC1-expressing cell line A375-CADM1/TSLC1 (A1) and empty vector-transfected control cell line A375-pcDNA3.1 (A0) were established by transfection with pcDNA-CADM1/TSLC1 or empty pcDNA3.1 vectors, respectively. An 3-(4,5-dimethylthiazol-2-yl)-2,5-diphenyl-tetrazolium bromide (MTT) assay, flow cytometry and transwell chambers were utilized to detect proliferation, invasion and cell apoptosis, respectively.

Metastasis is a complex process during which tumor cells become invasive, migrating away from the primary tumor and 
passing through natural extracellular matrix (ECM)-based barriers impeding access to vascular or lymphatic vessels.

Matrix metalloproteinase-2 (MMP-2) and matrix metalloproteinase-9 (MMP-9) are two important members in the metalloproteinase superfamily which are structurally related proteolytic enzymes that facilitate the degradation of ECM and the basement membrane. It has been demonstrated that MMPs are closely correlated with tumor invasion, since their upregulation markedly facilitated cancer cell migration through the ECM (9).

To further examine the possible anti-invasive mechanism of CADM1/TSLC1, the expression of MMP-2 and MMP-9 in A375 cells with different treatment were analyzed by western blotting.

\section{Materials and methods}

Cell line and culture. The human melanoma cell line A375 was provided by Tumor Institute of Harbin Medical University (Harbin, Heilongjiang, China). The cells were maintained in the RPMI-1640 (Beijing Solarbio Science \& Technology Co., Ltd., Beijing, China), supplemented with $10 \%$ fetal bovine serum (Gibco, Eggenstein, Germany), $100 \mathrm{U} / \mathrm{ml}$ of penicillin and $100 \mathrm{ug} / \mathrm{ml}$ of streptomycin at $37^{\circ} \mathrm{C}$ in a $5 \% \mathrm{CO}_{2}$ atmosphere.

Construction of plasmid expressing human CAMD1/TSLC1. Full length fragment of TSLC1 was amplified from RNA of hepatic cell L-02 by RT-PCR. The sequences of human CAMD1/TSLC1 primers were as follows: the forward primer, GACATGGCGAGTGTAGTGCT; the reverse primer, TGGGTCTGCAGGTTTCCAGT. PCR was performed using LA Taq System (TaKaRa Biotechnology (Dalian) Co., Ltd, Dalian, China) in 25 cycles of $94^{\circ} \mathrm{C}$ for $50 \mathrm{sec}, 58^{\circ} \mathrm{C}$ for $30 \mathrm{sec}$ and $72^{\circ} \mathrm{C}$ for $90 \mathrm{sec}$. PCR products were ligated into the pcDNA3.1 vector (Invitrogen Life Technologies, Carlsbad, CA, USA) to obtain the pcDNA-CADM1/TSLC1 vector.

Establishment of stable cell lines. The A375 cells were maintained in high glucose RPMI-1640 supplemented with $10 \%$ PBS, $100 \mathrm{U} / \mathrm{ml}$ penicillin and $100 \mu \mathrm{g} / \mathrm{ml}$ streptomycin. The A375 cells were transfected with pcDNA-CAMD1/TSLC1 and empty pcDNA3.1 vector, respectively, using Lipofectamine 2000 (Invitrogen Life Technologies) according to the manufacturer's instructions. The transfected cells were selected and maintained in full medium containing $600 \mu \mathrm{g} / \mathrm{ml} \mathrm{G} 418$ (Invitrogen Life Technologies). The cells stably expressing CAMD1/TSLC1 were then isolated and the expression of CAMD1/TSLC1 was confirmed by PCR and western blotting.

Western blotting analysis. Western blotting was conducted according to standard methods as described previously (8). Briefly, the cells were washed with PBS, then treated with a lysis buffer and protease inhibitor mixture on ice for $25 \mathrm{~min}$ and centrifuged. The protein samples were separated with 12\% SDS-PAGE and subsequently transferred onto PVDF membranes (Millipore, Billerica, MA, USA). The membranes were blocked with $5 \%$ skimmed milk solution in PBS for $60 \mathrm{~min}$ at room temperature, and incubated with rabbit anti-CADM1/TSLC1, MMP-2 and MMP-9 (Santa Cruz Biotechnology Inc., Santa Cruz, CA, USA), followed by bioti- nylated goat anti-rabbit IgG (Sigma, St. Louis, MO, USA) each for $2 \mathrm{~h}$ at $37^{\circ} \mathrm{C}$. The membranes were stained with an enhanced chemiluminescence solution (PerkinElmer, Boston, MA, USA). The images were acquired using an Image Quant350 digital image system (GE Healthcare, Uppsala, Sweden).

Cell proliferation analysis. To examine the cell proliferation, the cell growth of A375, A0 and A1 cells were analyzed by an MTT assay. Briefly, 1x10 cells were seeded 96-well plates and the cell growth was measured per $24 \mathrm{~h}$ until day 4 . A volume of $30 \mu \mathrm{l}$ of MTT (Sigma Chemical Co., St. Louis, MO, USA) solution $(5 \mathrm{mg} / \mathrm{ml})$ was then added and the cells were further incubated at $37^{\circ} \mathrm{C}$ for $4 \mathrm{~h}$. After $100 \mu \mathrm{l}$ of DMSO was added, the photodensity value at $540 \mathrm{~nm}$ was determined by an Opsys MR microplate reader (Thermo Labsystems, Beverly, MA, USA). Measurements of cell growth by the MTT assay were expressed as a percentage of the inhibition according to the following formula: Inhibition rate $(\%)=[(\mathrm{A}$ value of vector-alone transfectant - A value of CADM1 transfectant)/A value of vector-alone transfectant] x $100 \%$.

Cell migration assay. The effect of CADM1/TSLC1 on tumor cell invasion was investigated in vitro by an cell migration assay that was performed using 96-well plate transwell chambers (BD Biosciences, Franklin Lakes, NJ, USA) according to the manufacturer's instructions. In brief, $2 \times 10^{5}$ of $\mathrm{A} 375, \mathrm{~A} 0$ and A1 cells in $500 \mu \mathrm{l}$ serum-free medium were seeded into the upper part of each chamber of 96-well Matrigel chambers, respectively. A total of $24 \mathrm{~h}$ later, the cells were fixed and stained, and the number of cells on the lower surface of the filters was counted under the microscope (Olympus BX51; Olympus Corporation, Tokyo, Japan).

Cell apoptosis assay. For the apoptosis assay, $1 \times 10^{5} \mathrm{~A} 375, \mathrm{~A} 0$ and A1 cells were trypsinized at $48 \mathrm{~h}$, washed with cold PBS, and resuspended in PBS. Then, $10 \mu \mathrm{l}$ of Annexin V-FITC (BD Biosciences) and $5 \mathrm{ul}$ of propidium iodide (PI) were added. After the cells were vortexed and incubated for $15 \mathrm{~min}$ at room temperature, $400 \mu \mathrm{l}$ of binding buffer was added to the mixture. Flow cytometry was conducted on a FAC Scan instrument (BD Biosciences).

Statistical analyses. Statistical analysis was performed using SPSS version 13.0 (SPSS, Inc., Chicago, IL, USA). Summary results were described as the mean \pm standard error of the mean, and analysis of variance was used for the comparison of multiple means among the three groups. $\mathrm{P}<0.05$ was considered to indicate a statistically significant difference.

\section{Results}

CAMD1/TSLC1 expression in A375 cells with different treatment. To evaluate the biological role of CAMD1/TSLC1 in melanoma, we established stable CAMD1/TSLC1-expressing cell line A375-CAMD1/TSLC1 (A1) and empty vector-transfected control cell line A375-pcDNA3.1 (A0) by transfection with pcDNA-CAMD1/TSLC1 or empty pcDNA3.1 vectors, respectively. The results of RT-PCR and western blotting demonstrated that the level of CAMD1/TSLC1 mRNA (Fig. 1) and protein expression (Fig. 2) were significantly increased in 
Table I. Analysis of cell apoptosis by Annexin V/PI staining.

\begin{tabular}{|c|c|c|c|c|c|}
\hline Cell line & $\begin{array}{l}\text { Apoptotic } \\
\text { cell }(\%)\end{array}$ & $\begin{array}{c}\text { Late phase } \\
\text { apoptosis }(\%)\end{array}$ & $\begin{array}{l}\text { Living } \\
\text { cell }(\%)\end{array}$ & $\begin{array}{c}\text { Early phase } \\
\text { apoptosis }(\%)\end{array}$ & $\begin{array}{c}\text { Number of } \\
\text { apoptotic cells } \\
\text { (both phases) }\end{array}$ \\
\hline A1 & $0.59 \pm 0.01$ & $18.11 \pm 0.3^{\mathrm{a}}$ & $68.32 \pm 0.6$ & $16.59 \pm 0.1^{\mathrm{a}}$ & $37.6 \pm 0.1^{\mathrm{a}}$ \\
\hline A0 & $0.23 \pm 0.05$ & $2.27 \pm 0.1$ & $96.00 \pm 0.3$ & $1.53 \pm 0.3$ & $3.98 \pm 0.4$ \\
\hline A375 & $0.08 \pm 0.03$ & $3.36 \pm 0.2$ & $93.58 \pm 0.1$ & $2.58 \pm 0.5$ & $5.62 \pm 0.9$ \\
\hline
\end{tabular}

${ }^{\mathrm{a}} \mathrm{P}<0.01$ as compared with $\mathrm{A} 0$ and A357 cells. A0, A375-pcDNA3.1; A1, A375-CADMI/TSLC1; CADM1/TSLC1, cell adhesion molecule 1/tumor suppressor in lung cancer-1; PI, propidium iodide.

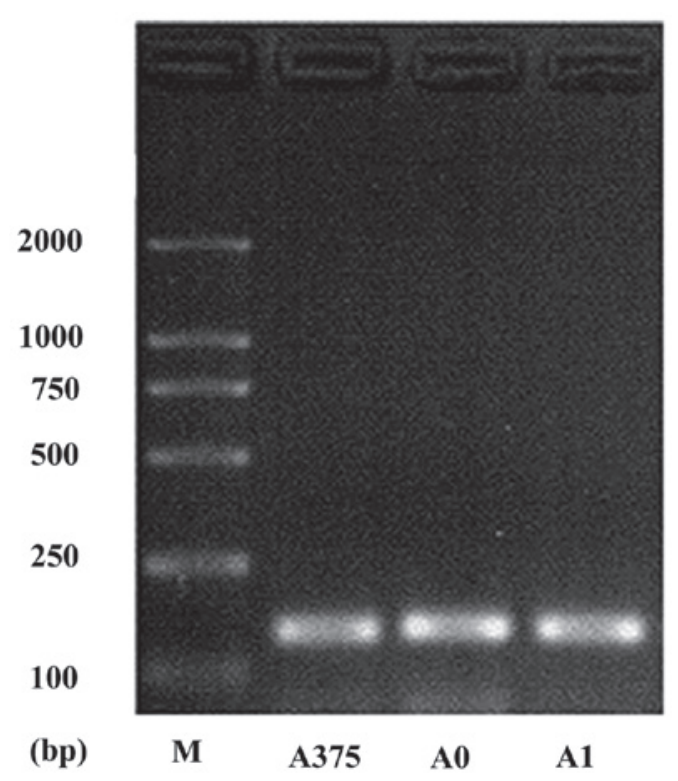

Figure 1. CADM1/TSLC1 mRNA expression. CADM1/TSLC1 mRNA expression levels were examined by RT-PCR. The expression level of the CADM1/TSLC1 gene was normalized to that of the housekeeping gene, $\beta$-actin. The relative levels of CADM1/TSLC1 mRNA in the three groups were counted using the formula $2 \Delta \Delta \mathrm{Ct}$. The values are expressed as the mean \pm standard error of the mean. Compared with A375 and A0, A1 demonstrated a higher level of CADM1/TSLC1 mRNA expression $(\mathrm{P}<0.05)$. A0, A375-pcDNA3.1; A1, A375-CADMI/TSLC1; CADM1/TSLC1, cell adhesion molecule 1/tumor suppressor in lung cancer-1.

A1 cells, compared with the A375 and A0 cells. However, there were no significant differences in the levels of CAMD1/TSLC1 mRNA and protein expression between the A375 cells and A0 cells $(\mathrm{P}>0.05)$.

Expression of CAMD1/TSLC1 inhibits cell proliferation. To study the effects of CAMD1/TSLC1 on cell growth, the cell viability of the transfected and non-transfected A375 cells was measured by an MTT assay. As demonstrated in Fig. 3, the growth of A1 clone was slower than that of A0 and A375 cells during the $96 \mathrm{~h}$ incubation period, but no significant differences between the A0 and A375 cells were observed ( $\mathrm{P}>0.05)$.

Expression of CADM1/TSLC1 induces apoptosis. To determine the apoptotic cell death in A375 cells induced by CAMD1/TSLC1, A375, A0 and A1 cells were stained with Annexin V/PI. As demonstrated in Fig. 3 and Table I, the results
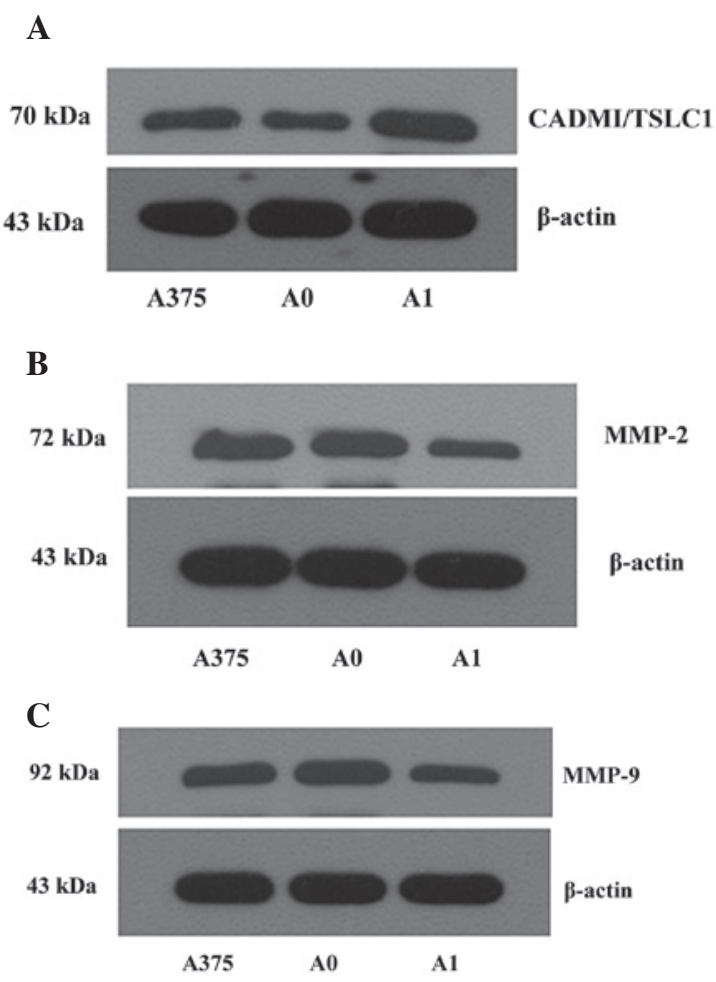

Figure 2. CADM1/TSLC1 and MMP-2, MMP-9 protein expression as determined by western blot analysis with an anti-CADM1/TSLC1 and MMP-2, MMP-9 antibody. The protein expression levels were semi-quantified by measuring the gray scale normalized to that of the housekeeping protein, $\beta$-actin. The relative protein expression levels were calculated by $\mathrm{CADMI} / \mathrm{TSLC} 1 / \beta$-actin ratios. The values are expressed as the mean $\pm \mathrm{stan}$ dard error of the mean. (A) Compared with A375 and A0, A1 demonstrated a higher level of CADM1/TSLC1 protein expression $(\mathrm{P}<0.05)$; $(\mathrm{B})$ compared with A375 and A0, A1 demonstrated a lower level of MMP-2 protein expression $(\mathrm{P}<0.05)$; $(\mathrm{C})$ compared with $\mathrm{A} 375$ and $\mathrm{A} 0, \mathrm{~A} 1$ demonstrated a lower level of MMP-9 protein expression $(\mathrm{P}<0.05)$. A0, A375-pcDNA3.1; A1, A375-CADMI/TSLC1; CADM1/TSLC1, cell adhesion molecule 1/tumor suppressor in lung cancer-1; MMP-2/9, matrix metalloproteinase-2/9.

of FACS analyses revealed that the proportion of positive cells were evidently increased in A1 cells $(37.60 \pm 0.1 \%$; $\mathrm{P}<0.01)$, compared with the A375 cells and A0 cells (3.98 \pm 0.4 and $5.62 \pm 0.9 \%$, respectively). Furthermore, there was a significant increase in the number of apoptotic cells both in the early phase and late phase in A375-TSLC1 cells as well. However, there was no difference in apoptosis rate between the A375 and A0 cells ( $\mathrm{P}>0.05$ ), indicating that the overexpression of CAMD1/TSLC1 has an inductive effect on apoptosis of A375 cells. 


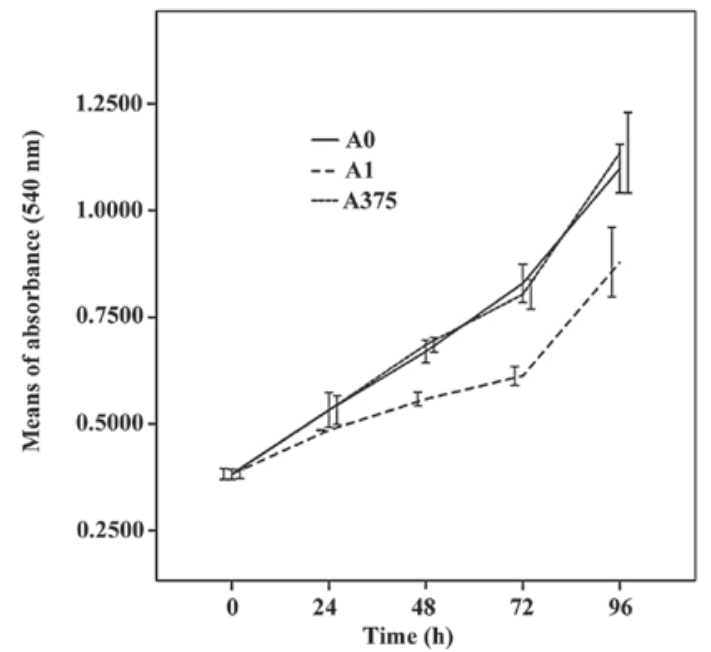

Figure 3. Effects of CADM1/TSLC1 on A375 cell proliferation as determined by an MTT assay. The value of absorbance (A540 $\mathrm{nm}$ ) was measured for proliferation analysis in the microplate reader. The values are expressed as the mean \pm standard error of the mean. Compared with A375 and A0, the growth of A1 clone was slower than that of A0 or A1 cells during the 96-h incubation period $(\mathrm{P}<0.05)$. A0, A375-pcDNA3.1; A1, A375-CADMI/TSLC1; CADM1/TSLC1, cell adhesion molecule 1/tumor suppressor in lung cancer-1; MTT, 3-(4,5-dimethylthiazol-2-yl)-2,5-diphenyl-tetrazolium bromide.

CADM1/TSLC1 suppresses cell migration. To investigate the effect of CAMD1/TSLC1 on cell metastasis, the cell migration ability of A375, A0 and A01 cells was examined by transwell assay. As demonstrated in Fig. 4, the cell migration assay revealed that the proportion of cells that transferring through the matrigel was significantly suppressed by $86.7 \%$ in A1 cells compared with that in the A0 (43.4\%) and A375 (25.8\%) cells. However, no significant differences between the A0 and A375 cells were observed in the transwell assay.

CADM1/TSLC1 downregulates MMP-2 and MMP-9 expression. To further investigate the molecular mechanisms involved in the effect of CADM1/TSLC1 overexpression on tumor cell invasion, the expression of MMP-2, -9, were determined by western blotting in A375, A0 and A1 cells. As demonstrated in Fig. 2B and C, the expression of MMP-2 and MMP-9 were significantly downregulated in the A1 cells, compared with that in the $\mathrm{A} 0$ and $\mathrm{A} 375$ cells.

\section{Discussion}

Melanoma is a malignant tumor of melanocytes that causes the majority of skin cancer-associated mortalities (10). The 5 -year survival rate decreases from $95 \%$ for patients with a maximum tumor thickness of $1 \mathrm{~mm}$ lacking metastases, to $<10 \%$ for patients with visceral metastasis. There is a conspicuous difference in survival between localized and metastatic disease (5-year survival of 98 and 15-62\%, respectively) (11). The occurrence of metastasis is associated with high mortality rates due to the aggressiveness characteristics of the tumor and the lack of effective therapies to combat its spread. Therefore, identifying the molecular mechanism underlying tumor progression and metastasis in cancer is urgently required.

CADM1, also known as TSLC1, as a novel tumor suppressor, has been extensively investigated in various tumors (12-16).
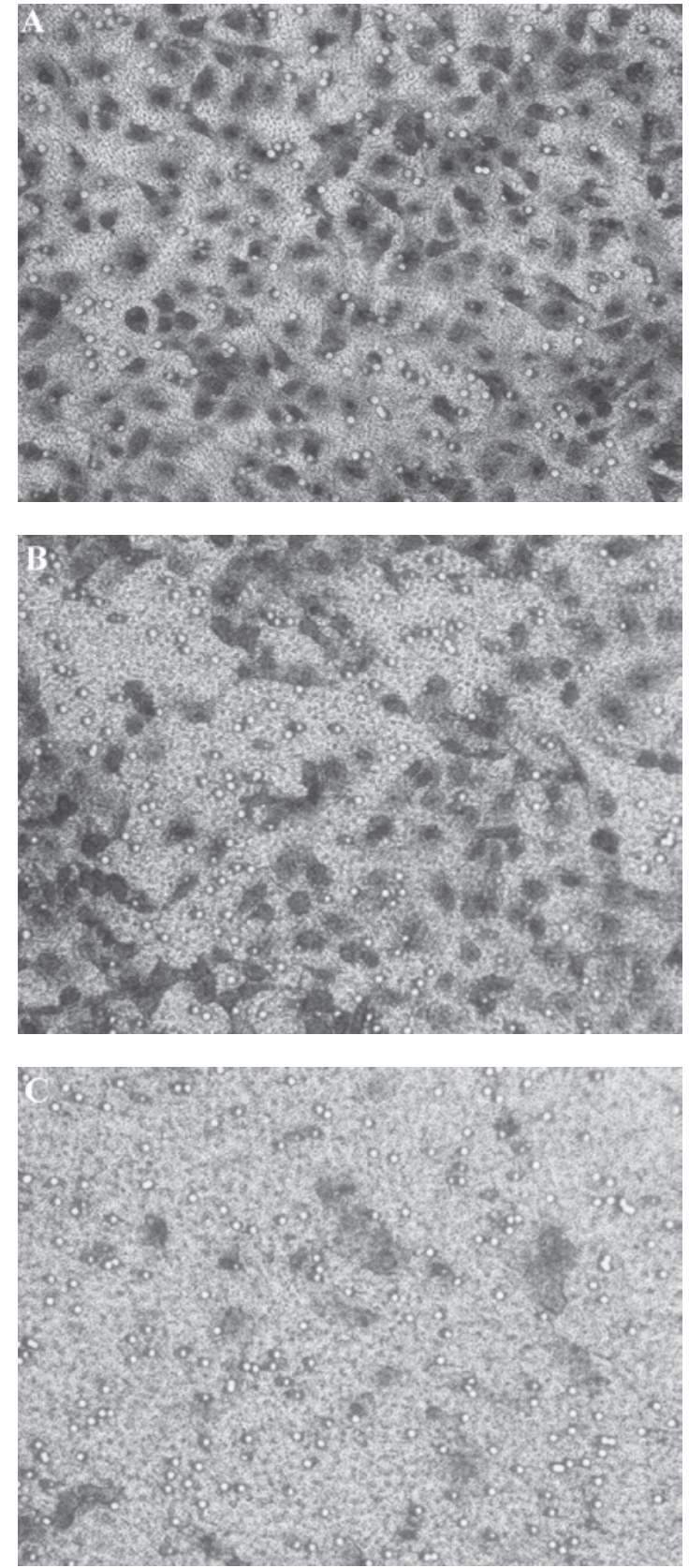

Figure 4. Effects of CADM1/TSLC1 on A375 cell invasion as determined by a transwell assay. All data were analyzed by SPSS 13.0 software, which was expressed as the mean \pm standard error of the mean. The invasive capacity was significantly suppressed in A1 cells compared with that in A0 and A375 cells $(\mathrm{P}<0.05)$. A0, A375-pcDNA3.1; A1, A375-CADMI/TSLC1; CADM1/TSLC1, cell adhesion molecule 1/tumor suppressor in lung cancer-1.

Loss or reduction of TSLC1 expression has been frequently found and demonstrated to be involved in the occurrence and progression of multiple different human tumors (17-20). Our previous results demonstrated that the silencing of TSLC1 through methylation is an important event in the pathogenesis of melanoma. Loss of the expression of TSLC1 in the cytoplasm of melanoma is associated with later tumor stage and decreased patient survival. TSLC1 thus constitutes a clinically important prognostic marker and a potential target for the development of novel therapies.

Accumulative evidence has revealed that CAMD1/TSLC1 is a crucial regulator of cell proliferation, invasion and 
apoptosis (21-23). In the present study, to verify the molecular mechanism of the tumor-suppressing effect of CAMD1/TSLC1 in melanoma, a A 375 cell line stably expressing CAMD1/TSLC1, A1, was successfully established and was used to investigate the role of CAMD1/TSLC1. In vitro, cell growth suppression by CAMD1/TSLC1 expression was demonstrated in that the cell proliferation was evidently inhibited in the A1 cells compared with the A375 and A0 cells $(\mathrm{P}<0.05)$, whereas there was no significant difference in the proliferation between the A375 and A0 cells ( $P>0.05)$. It was concluded that the inhibition of CAMD1/TSLC1 on cell invasion was, at least in part, due to the inhibition of proliferation.

It is well established that apoptosis is an important physiological process responsible for maintaining the balance of homeostasis and that it has a central role in the progression and development of tumors. In the present study, Annexin V/PI staining demonstrated a significant increase in the number of total apoptotic cells of A1 other than in A375 and A0 cells. This indicated that the overexpression of CAMD1/TSLC1 has an inductive effect on the apoptosis of A375 cells.

Malignant tumor invasion is a dynamic, continuous process. Tumor cells migrate away from the primary site, first invading the extracellular matrix (ECM) and the basement membrane and the interstitial cells in some molecular adhesion, and then activating cell synthesis and the secretion of various degradation enzymes, that assist in the migration of tumor cells through the ECM into the blood vessels. Then under the regulation of certain factors, including running through the vessel wall leakage to the secondary site, they continue to proliferate and subsequently result in the formation of metastases with peeling, adhesion, degradation, mobility, proliferation and transfer throughout the course of malignant tumor invasion.

In the present study, to determine whether the invasion ability of the A375 cells, matrigel, which simulates the metastatic process of tumor cells to travel through the ECM and basement membrane components, was inhibited, the cell migration ability of A375, A0 and A01 cells was examined by a transwell assay. As demonstrated in Fig. 4, the transwell assay revealed that the invasive capacity was significantly suppressed in A1 cells compared with that in the A0 and A375 cells , but no significant differences between the A0 and A375 cells were observed, which indicated that the overexpression of CAMD1/TSLC1 had an inductive effect on the migration ability of melanoma.

MMPs are a family of structurally related proteolytic enzymes that facilitate the degradation of ECM and the basement membrane. MMP-2 and MMP-9 are two important members in the metalloproteinase superfamily, which are involved in a wide range of proteolytic events, including tumor growth, migration, metastasis and angiogenesis (24). In the present study, to further examine the probable anti-invasive mechanism of CAMD1/TSLC1, the expression of MMP-9 and MMP-2 in A375, A0 and A1 cells was examined. As is demonstrated in Fig. 2., the A1 cells revealed a marked expression of MMP-9 and MMP-2. Therefore, it was hypothesized that the invasion inhibitory effect of CAMD1/TSLC1 may be due to the suppression of MMP-9 and MMP-2 expression, which subsequently prompts tumor metastasis and invasion. The details of the mechanism involved required further validation.
In conclusion, the present study demonstrated that CADM1/TSLC1 had anti-invasive effects on A375 cells. This inhibition was correlated with the expressional downregulation of MMP-2and MMP-9, which are associated with tumor metastasis and progression.

\section{Acknowledgements}

This study was supported by the Foundation of Technique of Heilongjiang Province, China (no. C201317). The authors would like to thank Xiwen Sun for providing valuable suggestions and assistance and Yuyan Ma for his excellent technical assistance.

\section{References}

1. Kuramochi M, Fukuhara H, Nobukuni T, et al: TSLC1 is a tumor suppressor gene in human non-small cell lung cancer. Nat Genet 27: 427-430, 2001

2. Uchino K, Ito A, Wakayama T, et al: Clinical implication and prognostic significance of the tumor suppressor TSLC1 gene detected in adenocarcinoma of the lung. Cancer 98: 1002-1007, 2003.

3. Tamura G: Promoter methylation status of tumor suppressor and Tumor related genes in neoplastic and non-neoplastic gastric epithelia. Histol Histopathol 19: 221-228,2004.

4. Dewan MZ, Takamatsu N, Hidaka T, et al: Critical role for TSLC1 expression in the growth and organ infiltration of adult T-cell leukemia cells in vivo. J Virol 82: 11958-11963, 2008.

5. Yang G, He W, Cai M, et al: Loss/Down-regulation of tumor suppressor in lung cancer 1 expression is associated with tumor progression and is a biomarker of poor prognosis in ovarian carcinoma. Int J Gynecol Cancer 21: 486-493, 2011.

6. Jansen M, Fukushima N, Rosty C, et al: Aberrant methylation of the 5-CpG island of TSLC1 is common in pancreatic ductal adenocarcinoma and is first manifest in high-grade PanlNs. Cancer Biol Ther 1: 293-296, 2002

7. Crawford NP, Qian X, Ziogas A, et al: Rrplb, a new candidate susceptibility gene for breast cancer progression and metastasis. PLoS Genet 3: e214, 2007

8. You Y, Ma L, You M, et al: TSLC1 gene silencing in cutaneous melanoma. Melanoma Res 20: 179-183, 2010.

9. Herszényi L, Hritz I, Lakatos G, Varga MZ and Tulassay Z: The behavior of matrix metalloproteinases and their inhibitors in colorectal cancer. Int J Mol Sci 13: 13240-13263, 2012.

10. Meyskens FL Jr, Farmer PJ, Yang S and Anton-Culver H: New perspectives on melanoma pathogenesis and chemoprevention. Recent Results Cancer Res 174: 191-195, 2007.

11. Balch CM, Buzaid AC, Soong SJ, et al: Final version of the American Joint Committee on Cancer staging system for cutaneous melanoma. J Clin Oncol 19: 3635-3648, 2001.

12. Takahashi Y, Iwai M, Kawai T, et al: Aberrant expression of tumor suppressors CADM1 and 4.1B in invasive lesions of primary breast cancer. Breast Cancer 19: 242-252, 2012.

13. Heller G, Fong KM, Girard L, et al: Expression and methylation pattern of TSLC1 cascade genes in lung carcinomas. Oncogene 25: 959-968, 2006.

14. Houshmandi SS, Surace EI, Zhang HB, et al: Tumor suppressor in lung cancer-1 (TSLC1) functions as a glioma tumor suppressor. Neurology 67: 1863-1866, 2006.

15. Fukami T, Fukuhara H, Kuramochi M, et al: Promoter methylation of the TSLC1 gene in advanced lung tumors and various cancer cell lines. Int J Cancer 107: 53-59, 2003.

16. Yamada D, Yoshida M, Williams YN, et al: Disruption of spermatogenic cell adhesion and male infertility in mice lacking TSLC1/IGSF4, an immunoglobulin superfamily cell adhesion molecule. Mol Cell Biol 26: 3610-3624, 2006.

17. He G, Lei W, Wang S, et al: Overexpression of tumor suppressor TSLC1 by a survivin-regulated oncolytic adenovirus significantly inhibits hepatocellular carcinoma growth. J Cancer Res Clin Oncol 138: 657-670, 2012.

18. Surace EI, Lusis E, Murakami Y, et al: Loss of tumor suppressor in lung cancer-1 (TSLC1) expression in meningioma correlates with increased malignancy grade and reduced patient survival. J Neuropathol Exp Neurol 63: 1015-1027, 2004. 
19. Chen K, Wang G, Peng L, et al: CADM1/TSLC1 inactivation by promoter hypermethylation is a frequent event in colorectal carcinogenesis and correlates with late stages of the disease. Int J Cancer 128: 266-273, 2011.

20. Ando K, Ohira M, Ozaki T, et al: Expression of TSLC1, a candidate tumor suppressor gene mapped to chromosome $11 \mathrm{q} 23$, is downregulated in unfavorable neuroblastoma without promoter hypermethylation. Int J Cancer 123: 2087-2094, 2008.

21. Sussan TE, Pletcher MT, Murakami Y and Reeves RH. Tumor suppressor in lung cancer 1 (TSLC1) alters tumorigenic growth properties and gene expression. Mol Cancer 4: 28, 2005.
22. Qin L, Zhu W, Xu T, et al: Effect of TSLC1 gene on proliferation, invasion and apoptosis of human hepatocellular carcinoma cell line HepG2. J Huazhong Univ Sci Technolog Med Sci 27: 535-537, 2007.

23. Mao X, Seidlitz E, Truant R, Hitt M and Ghosh HP: Re-expression of TSLC1 in a non-small-cell lung cancer cell line induces apoptosis and inhibits tumor growth. Oncogene 23: 5632-5642, 2004.

24. Giannelli G and Antonaci S: MMP and TIMP assay in cancer: Biological and clinical significance. Int J Cancer 116: 1002-1003, 2005. 